\title{
Validity of the British system for anticoagulant control using the national reagent
}

\author{
M. R. AlderSON, L. POLlER, AND JEAN M. THOMSON \\ From the Department of Social and Preventive Medicine, University of Manchester, and the \\ Haematology Department, Withington Hospital, Manchester
}

SYNOPSIS The national system for anticoagulant control depends on drawing the best $\frac{}{3}$ line obtained by visual comparison of the points representing corresponding prothrombin $\vec{z}$ ratios with the British comparative thromboplastin in the local reagent. This line is then used to correct subsequent values using the local laboratory method of corresponding values in $\vec{\theta}$ terms of the British comparative thromboplastin.

A study has been made of the statistical validity of the recommended system for anticoagulant control using the Quick one-stage test by comparing the line drawn by inspection with the confidence limits of the regression line.

There was little difference between the best straight line by visual comparison and the calcu- $\frac{\varrho}{\triangleright}$ lated line for the majority of hospitals. The recommended procedure, therefore, provides an adequate conversion of the local method to the British comparative thromboplastin without $\stackrel{?}{3}$ the need for calculation.

The significance of aberrant points is discussed and it is suggested that when more than two of the 12 results fall in this category the standardization procedure should be repeated.

A national scheme for anticoagulant control has been introduced recently by the British Committee for Standards in Haematology. This is based on two principles: (1) the use of a: single tissue extract, the British comparative thromboplastin, known formerly as the Manchester comparative reagent, as a reference reagent in a standardization procedure (ACP Broadsheet, in press). The BCT is used to characterize 'home-made' and commercial reagents; (2) the use of a uniform system of reporting prothrombin results, ie, the British corrected ratio. At each hospital where the British comparative thromboplastin is used the prothrombin ratio results determined on at least 12 patients with the usual laboratory method, are compared with those obtained in parallel with the reference preparation. Each patient's results are plotted Received for publication 11 February 1970. on graph paper to give a visual comparison of $\frac{D}{9}$ the results with the two techniques. Following inspection of the points on the graph, a line is $N$ then drawn which is thought to be the "best $N$ straight line' representation of the relationship ${ }_{N}$ between the two techniques. This line is then $\omega$ used to convert subsequent values obtained using the usual laboratory method to corresponding $\stackrel{\circ}{\subset}$

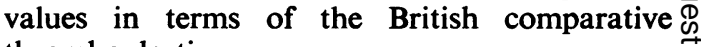
thromboplastin.

There is a mathematical method for fitting $\frac{T}{0}$ a line to such data using the conventional $\underset{D}{\mathbb{D}}$ formula for linear regression analysis, but as $\frac{?}{\mathbb{D}}$ such statistical calculations are not always $\varrho$ suitable for routine hospital laboratories, the simple system of visual comparison has been 8 recommended. Therefore, a study has been? made of the statistical validity of the recommended British system for anticoagulant control 
using the Quick one-stage test by comparing the line drawn by inspection with the confidence limits of the regression line.

\section{Method of Study}

Thirty hospitals were asked to participate in the study. The hospitals selected were all familiar with the British comparative thromboplastin and used the Quick test procedure routinely. They had all participated in our previous prothrombin time survey (Poller and Thomson, 1969) and had returned accurate and complete results. All received samples of the same batch of British comparative thromboplastin. The first group of 15 hospitals were sent samples of the same numbered batch of a phenolised commercial rabbit brain thromboplastin (Diagen). The other group of 15 hospitals were asked to compare the British comparative thromboplastin with their 'home-made' human brain tissue extract. The standardization procedure was as follows:

Hospitals were asked to test the two types of reagents in parallel. Nine vol of venous blood was added to $1 \mathrm{vol}$ of $3.13 \%$ tri-sodium citrate in a plastic or siliconed container and kept at $4^{\circ} \mathrm{C}$. Specimens were collected from two normal controls and 20 anticoagulated patients. To reduce the effect of contact activation hospitals were asked to test each plasma specimen in turn with both sets of reagents. They were asked to report prothrombin times in seconds on an accompanying sheet. We performed the subsequent calculations of prothrombin ratios. Then we plotted the results on arithmetical graph paper and drew the 'best straight line' between all the points by visual assessment. ${ }^{1}$ Linear regression analysis was carried out and the calculated line inserted on the same graph. The two lines were then compared for each centre and analysed to see if there was a significant difference between the line drawn by visual comparison and the calculated line in both groups of hospitals. The $95 \%$ confidence limits for the calculated line can be readily obtained; that is, limits of the displacement of the line that might occur by chance. The graphs were inspected to see

\footnotetext{
${ }^{1}$ Theoretically, it might be preferable that double log paper be used instead of arithmetic graph paper for plotting the results due to the wider scatter of results that might be expected with higher ratios. However, a comparison of the fitted regression lines with their confidence limits for the raw data and $\log$ values showed that there is little difference between the results with either type of graph paper; it is perfectly satisfactory, therefore, to use plain graph paper and somewhat simpler. The line that fits all the points best should be plotted whether these points are within the therapeutic range in terms of British corrected ratio or not. It will be found simplest to use a clear plastic ruler so that all the points may be observed whilst lining up the edge of the ruler with the line that appears to fit best these points. No attempt should be made to force the line through the origin of the graph. One might expect the line to go through the origin, but the line drawn should be the best representation of the values obtained in the laboratory, and for this purpose the origin may be ignored.
}

if the visual line fell outside these confidence limits.

The difference between the 'best straight line and the calculated line can be assessed bo measuring the discrepancy between the two lines at various points. Seven points with the following values on the scale of the local method were usecti $1 \cdot 8,2 \cdot 0,2 \cdot 2,2 \cdot 4,2 \cdot 6,2 \cdot 8$, and $3 \cdot 0$; in additiof the vertical distance between the two lines wos measured from the points on the calculated line equivalent to 1.8 and 3.0 with the Britis comparative thromboplastin. The latter two points are the extremes of the therapeutic scale with the Manchester reagent (now the Britisb comparative thromboplastin). At each of these points the discrepancy between the value fog the British corrected ratio provided by the calculated line and the line drawn followirg inspection has been measured.

\section{Results}

ANALYSIS OF RETURNS Of the 15 hospitals in the first group which were sent animal brain three were excluded. Ore centre was excluded because the British cons parative thromboplastin had been incorrect stored and become inactivated; the two oth $\overline{\overline{\mathrm{er}}}$ centres were unable to provide results. Of time 15 hospitals in the group which tested time 'home-made' human brain against the Britisiti comparative thromboplastin five were exclude $\mathbf{\text { s. }}$ Four centres were not able to provide da翯 for sufficient patients (two provided data fi़्r only 10 patients, one for eight, and one five); the fifth centre failed to provide values for normals. One hospital which had not been originally included in this group returned a corinplete set of results whilst the study was in progress and therefore was included.

There was little difference between the 'bes straight line' drawn through the points by vist comparison and the calculated line for the majority of the hospitals in both groups. Whese the two lines did not coincide, two basic patterios emerged (Figs. 1 and 2). The lines either divergof or crossed over from each other, confirmiffy the need to take multiple points in calculatimg the relationship between the calculated afd best line from visual estimation. The Tab indicates the mean discrepancy between the lines for each centre cooperating in the stugy for the seven points on the local scale afd the extremes of the therapeutic range in terfiss of British comparative thromboplastin. It w w be seen that there is a tendency for grear discrepancy with the animal brain compargd with hospitals in group 1 which used only hum brain. In three centres in group 1 and two cent in group 2 the visual line lay outside the con 


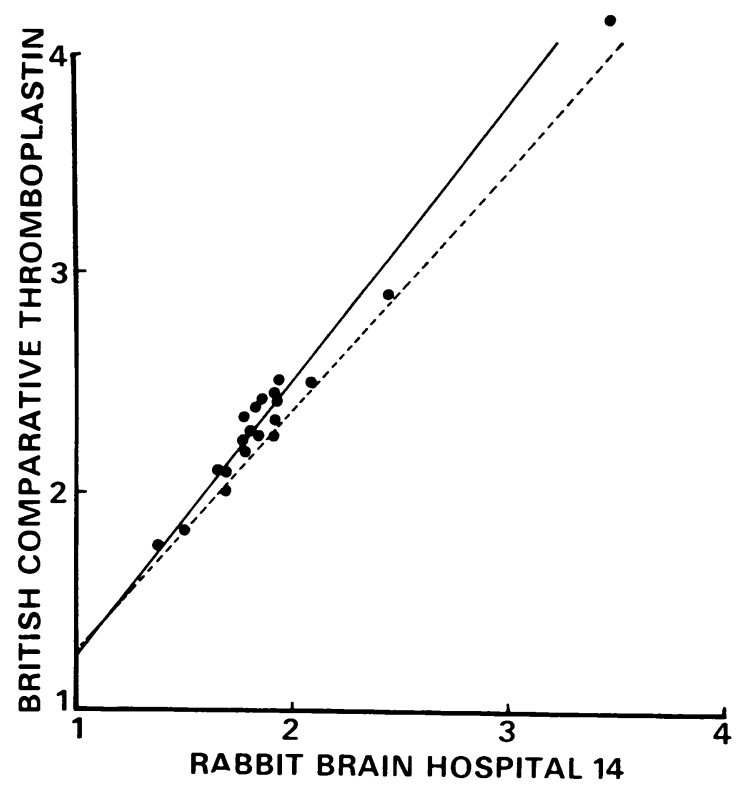

Fig. 1 Example of divergence between calculated and best straight line.

Calculated line - - - - -

Best straight line-

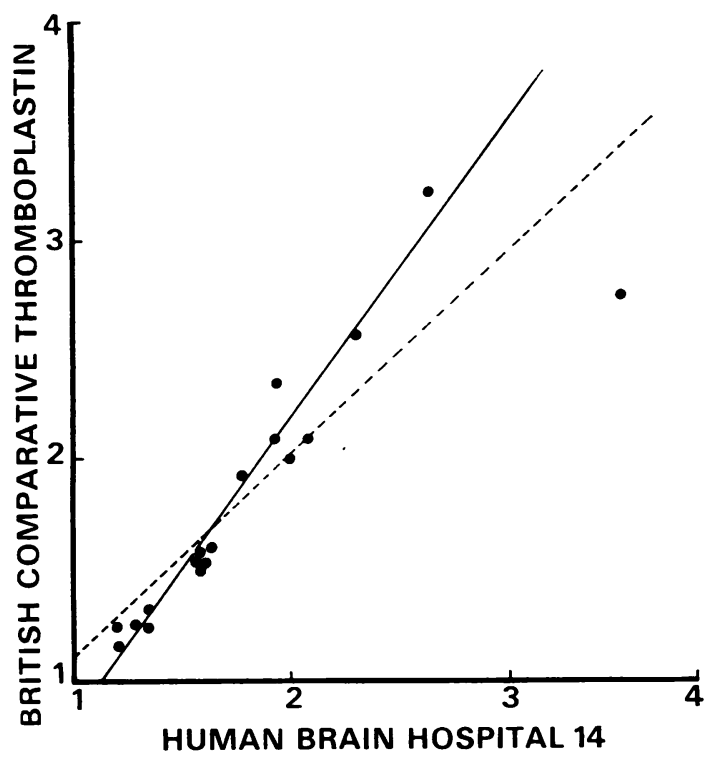

Fig. 2 Example of cross-over between calculated and best straight line.

dence limits of the calculated line. This was where the mean difference between the two lines was relatively high and the line has been drawn from results which included a considerable number of aberrant points, an insufficient number of points, or where results have been unevenly spread owing to a concentration of the points at one or other end of the scale. The

\begin{tabular}{ccl}
\hline Hospital Number & $\begin{array}{l}\text { Mean Discrepancy } \\
\text { for } 7 \text { Values on } \\
\text { Local Scale }\end{array}$ & $\begin{array}{l}\text { Mean Discrepancy } \\
\text { at } 1 \cdot 8 \text { and } 3.0 \\
\text { British Corrected } \\
\text { Ratios }\end{array}$ \\
\hline Local Human Brain $\mathbf{V}$ British Comparative Thromboplastin \\
1 & 0.05 & 0.04 \\
2 & 0.09 & 0.07 \\
3 & 0.02 & 0.02 \\
4 & 0.27 & 0.35 \\
5 & 0.17 & 0.18 \\
6 & 0.14 & 0.03 \\
7 & 0.11 & 0.17 \\
8 & 0.06 & 0.05 \\
9 & 0.14 & 0.04 \\
10 & 0.04 & 0.06 \\
11 & 0.06 & 0.08 \\
& & \\
Animal Brain v British Comparative & Thromboplastin \\
12 & 0.22 & 0.08 \\
13 & 0.91 & 0.25 \\
14 & 0.20 & 0.13 \\
15 & 0.20 & 0.13 \\
16 & 0.32 & 0.18 \\
17 & 0.11 & 0.06 \\
18 & 0.23 & 0.10 \\
19 & 0.06 & 0.06 \\
20 & 0.22 & 0.10 \\
21 & 0.20 & 0.35 \\
22 & 0.29 & 0.26 \\
23 & 0.08 & 0.05 \\
\hline
\end{tabular}

Table Mean discrepancy between lines for each centre for the seven points on the local scale and extremes'of therapeutic scale in terms of the British comparative thromboplastin

human brain group showed a greater tendency for the results to be close to the line and aberrant points were less frequent than in the animal brain group. In one of the five hospitals where the results were outside the confidence limits the elimination of the single 'rogue' point present in the results brought the line within the limits of confidence.

\section{Discussion}

The main conclusion from this study is that the anticoagulant dosage in any individual patient would not have been appreciably different at any of the hospitals if the British corrected ratio had been obtained from the best straight line or the calculated line. It is, therefore, unnecessary to calculate regression lines when comparing the British comparative thromboplastin with human brain and animal brain.

The calculation for the determination of the regression lines, although not too complicated, is obviously not simple and may be unsuited for routine pathology and haematology laboratories. To base a procedure to be used in all hospital laboratories on a calculation which it might not be possible for them to perform would therefore be undesirable. The fact that this calculation is seen to be unnecessary is reassuring.

From the practical and theoretical standpoint the line drawn by visual comparison is more 


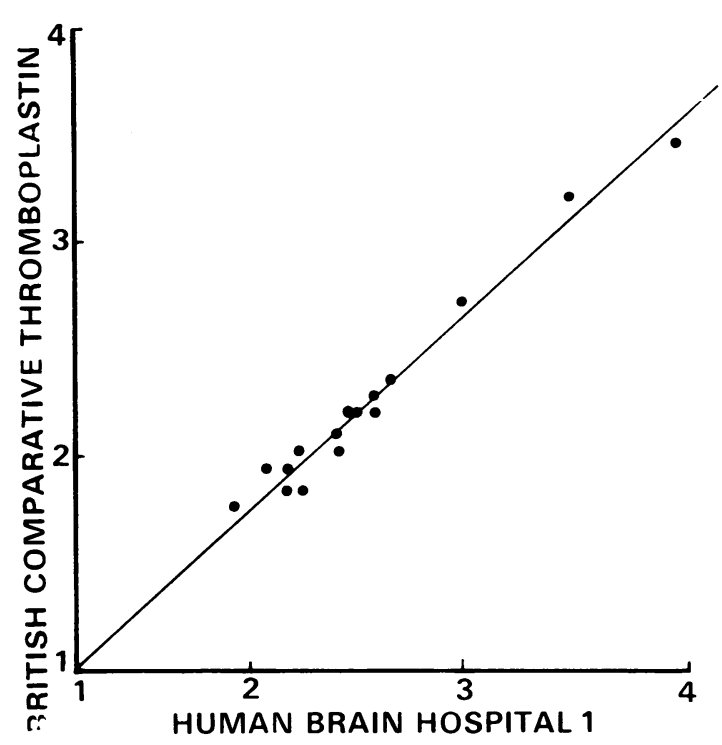

Fig. 3 Linear distribution of points.

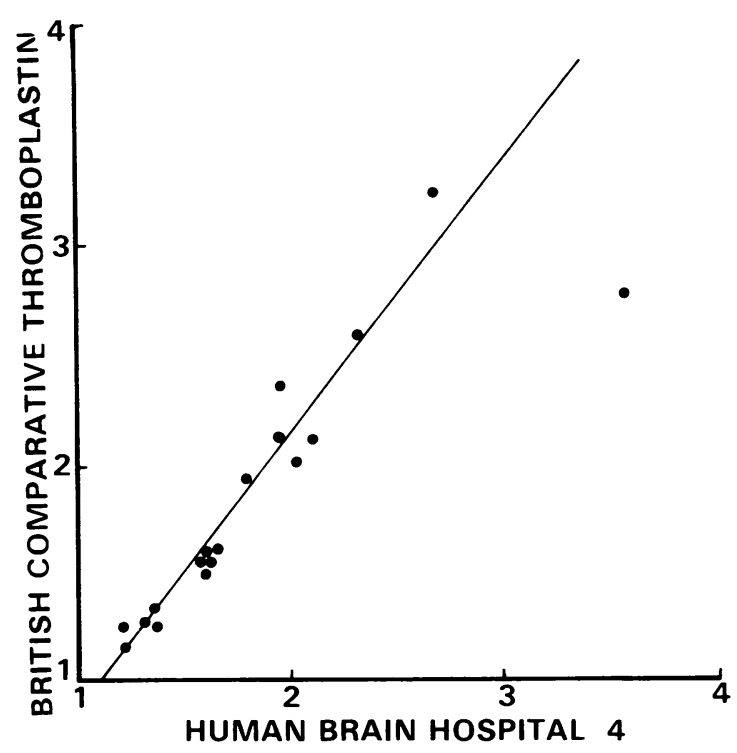

Fig. 5 Single aberrant points.

realistic in clinical practice than the line obtained by calculation. Drawing a line by visual assessment through points on a graph can be fairly simple if the points fall close to a linear distribution (Fig. 3). If there are a number of aberrant points the line drawn by visual means can vary considerably (Fig. 4). We have shown that when the British comparative thromboplastin is compared with human brain and animal brain extracts the line of 'best fit' is sufficiently close

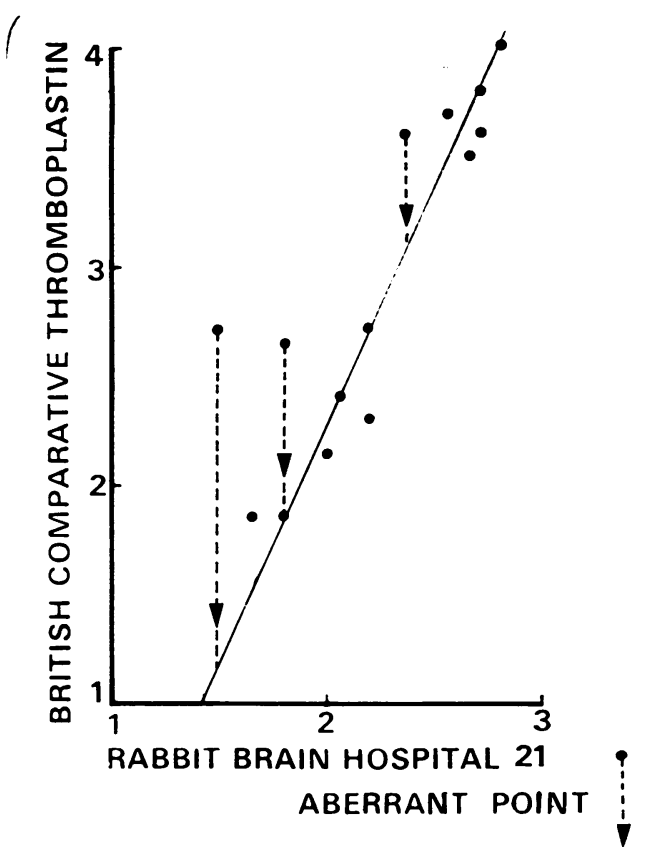

Fig. 4 Multiple aberrant points.

to the calculated line. Where discrepancies ariseis when a number of 'rogue' or 'aberrant' points occur (Fig. 4). When drawing the line visually, occasional aberrant points tend to be ignored, in order to produce a line which repre-aे sents most of the points, whereas the calculation $\frac{}{D}$ takes these aberrant points into account. This means it is less likely that the lines will coincide.

It is probably sound practice to ignore isolated aberrant points. A considerable number of variables may lead to disagreement between. successive tests on coumarin blood samples응 Thromboplastin reagents vary in sensitivityo to coumarin blood factors, and animal reagents are relatively insensitive to factors VII and X.̊ In the first few days of anticoagulant treatment factor VII may be the principal factor depressed 0 Although we asked hospitals to exclude patients who had been on anticoagulants for less than? 72 hours, occasionally a predominantly factor VII deficiency is present at that time, or even? later. In these circumstances the prothrombinf ratio with a reagent relatively insensitive tow factor VII will be much lower than the expectedb ratio corresponding to the British comparative thromboplastin and will be an aberrant result $\frac{\mathrm{D}}{\mathrm{D}}$ That this has occurred in this study is suggested? by the fact that although the 'best line' waso drawn without difficulty at all centres usinge Diagen, more aberrant points were presenf than at the centres using human brain. InD addition, many variables influence prothrombintimes and some of these are difficult to trace to explain an aberrant result. The following have been shown to cause discrepancies between consecutive prothrombin time tests:

(1) Collection of specimen: some thrombo 
plastin reagents are more affected by contact activation than others.

(2) Test tubes: discrepancies arise from incompletely washed or rinsed, and scratched tubes, variations in tube size and diameter.

(3) Faulty technique: errors arise from incorrect volumes of reagents and variable pipetting technique; variable warming of test tubes and reagents; variable periods of incubation and contact between thromboplastin and plasma.

An example of this can be seen in Fig. 5 where all the points are distributed on or close to a line with the exception of a single 'rogue' value. It is justifiable to plot the line through the remainder of the points, ignoring the single 'rogue' value; this should only be done where there are one or two outlying values with the remainder of the points closer to the line. Where more than two of the points of the 12 results on coumarin patients returned for the standardization procedure (ACP Broadsheet) are farther than 0.5 British corrected ratio from the line (in a vertical plane) it is suggested that the procedure be regarded as unsatisfactory, and it should be repeated with a fresh set of specimens. Figure 4 illustrates the results where a repeat of the procedure would be advisable. Unsatisfactory results may be caused by an unfortunate selection of specimens, eg, the inclusion of specimens with predominantly factor VII defects. Faulty technique (see above) will likewise result in aberrant points.

It is, of course more likely that a cluster of results in the higher ratio range will tend to give more rogue values than a similar group at the lower end of the ratio scale. The Committee has guarded against this by recommending that the 12 coumarin plasmas needed for the standardization procedure should fall within the range of 1.7 to 3.8 prothrombin ratio with the British comparative thromboplastin (ACP Broadsheet).

The authors wish to acknowledge the important part played in this study by the many participating laboratories in Britain. The British system for $\omega$ anticoagulant control has been evolved as a result of their continued cooperation and assistance.

Refereaces

The Use of the National Thromboplastin Reagent for Uniformity of Laboratory Control of Oral Anticoagulants and $\bar{z}$ Expression of Results. A.C.P. Broadsheet-in press.

Poller, L., and Thomson, Jean M. (1969). The interpretation of prothrombin results. A national survey. Brit. J. Haemat., 16,31 . 